University of Nebraska - Lincoln

DigitalCommons@University of Nebraska - Lincoln

Robert G. Fuller Publications and Presentations Research Papers in Physics and Astronomy

2-1-1977

Can physics develop reasoning?

Robert Fuller

rfuller@neb.rr.com

Robert Karplus

Lawrence Hall of Science and President of the American Association of Physics Teachers

Anton E. Lawson

University of California, Berkeley, anton.lawson@asu.edu

Follow this and additional works at: https://digitalcommons.unl.edu/physicsfuller

Part of the Physics Commons

Fuller, Robert; Karplus, Robert; and Lawson, Anton E., "Can physics develop reasoning?" (1977). Robert G. Fuller Publications and Presentations. 31.

https://digitalcommons.unl.edu/physicsfuller/31

This Article is brought to you for free and open access by the Research Papers in Physics and Astronomy at DigitalCommons@University of Nebraska - Lincoln. It has been accepted for inclusion in Robert G. Fuller Publications and Presentations by an authorized administrator of DigitalCommons@University of Nebraska - Lincoln. 


\title{
Can physics develop reasoning?
}

\author{
The findings of Swiss scholar Jean Piaget suggest that it can- \\ by helping people achieve a series of four distinct but overlapping stages \\ of intellectual arowth as thev search for Datterns and relationships.
}

\author{
Robert G. Fuller, Robert Karplus and Anton E. Lawson
}

The life of every physicist is punctuated by events that lead him to discover that the way physicists see natural phenomena is different from the way nonphysicists see them. Certain patterns of reasoning appear to be more common among physicists than in other groups. These include:

- focussing on the important variables (such as the force that accelerates the apple, rather than the lump it makes on your head);

- propositional logic ("if heat were a liquid it would occupy space and a cannorr barrel could only contain a limited amount of heat, but this is contrary to my observations, so ..."), and

- proportional reasoning (for example, the restoring force of a spring increases linearly with its displacement from equilibrium).

In recent studies of the reasoning used by students we bave discovered among them qualitative differences similar to those between the reasoning patterns of physicists and nonphysicists.

How can we understand these qualitative differences in reasoning? What role does physics play in the way reasoning develops in young people?

Along with a group of teachers in physics and other disciplines, we believe that some of the answers to these questions can be found in the work of developmental psychologists, especially that of

Robert G. Fuller is a visiting professor of physics at the University of California, Berkeley and a research physicist at the Lawrence Hall of Science while on leave from the University of Nebraska-Lincoln, where he is a professor of physics; Robert Karplus is the acting director of the Lawrence Hall of Science and President of the American Association of Physics Teachers, and Anton E. Lawson is a research associate at the Lawrence Hall of Science. University of California, Berkeley. the Swiss scholar Jean Piaget. We have helped start a modest movement, accordingly, to inform others of the relevant findings and theories of these social scientists.

To do so we have extended the psychologists' original investigations by dealing with their implications for the presentation of subject matter at the secondary-school and college levels. Textbooks, laboratory procedures, homework assignments, test questions and films may all be examined from the developmental point of view. ${ }^{1}$

In this article we shall describe those ideas in Piaget's work that we have found most useful; you may judge for yourself how valid they are. We shall conclude by suggesting ways in which you can use your expertise in physics and your personal contacts - whether you teach physics or not- to encourage others to develop their reasoning through their observations and analyses of physical systems.

\section{Student responses to puzzles}

To study the differences in reasoning used by students, we have devised a number of paper-and-pencil puzales and given them to high-school and college students. Let us examine the following typical student responses to two of these, the Ticker-Tape Puzzle and the Islands Puzzle, ${ }^{2}$ and discuss the differences in reasoning displayed in them by the students.

The responses to the Ticker-Tape Puzzle (see the Box on page 25) were collected from engineering and science students in an introductory physics course. Some of them had completed the term covering newtonian mechanics, others had not. Here are samples:

Fred (had used ticker tape)

1 B-Dots are spaced equally.

$2 \mathrm{C}$-Dots are closing together, cart is going less distance in the same time.

3 A-Dots are getting farther apart, cart is moving farther in same time (accelerating).

4 D-Cart is falling through air; it has a rapid acceleration.

James (had not used ticker tape)

I B-At constant speed, the same distance will be covered per unit time.

2 E-Deceleration means less velocity, so less distance per unit time.

3 D-Acceleration is exponential, ruling out A.

4 C-Assume a frictionless system, with brakes momentarily applied between dots five and six.

The responses to the Islands Puzzle (see the Box on page 26) were collected from a wide variety of adolescents and adults. These two are typical:

Deloris (College student, age 17)

1 "Yes, because the people can go north from Island D-because in the clue it could be made in both directions."

2 "No; I am presuming both directions doesn't include a $45^{\circ}$ angle from $B$ to c."

3 "Yes, because Island $\mathrm{C}$ is right below Island A."

Myrna (College student, age 17)

1 "Can't tell from the clues given. The two clues don't relate the upper islands to the lower ones."

2 "Yes; they can go from B to D, and then to $\mathrm{C}$, even if there are no direct flights."

3 "No, if they could go from $C$ to $A$, then the people on $B$ could go first to $D$, then to $C$, and then on to $A$. But this contradicts the second clue, that they don't go by plane between B and A."

You will notice some similarities between the responses of Fred (to the Ticker-Tape Puzzle) and Deloris (to the Island Puzzle). They both focus on the specific details of the puzzle. Fred makes 


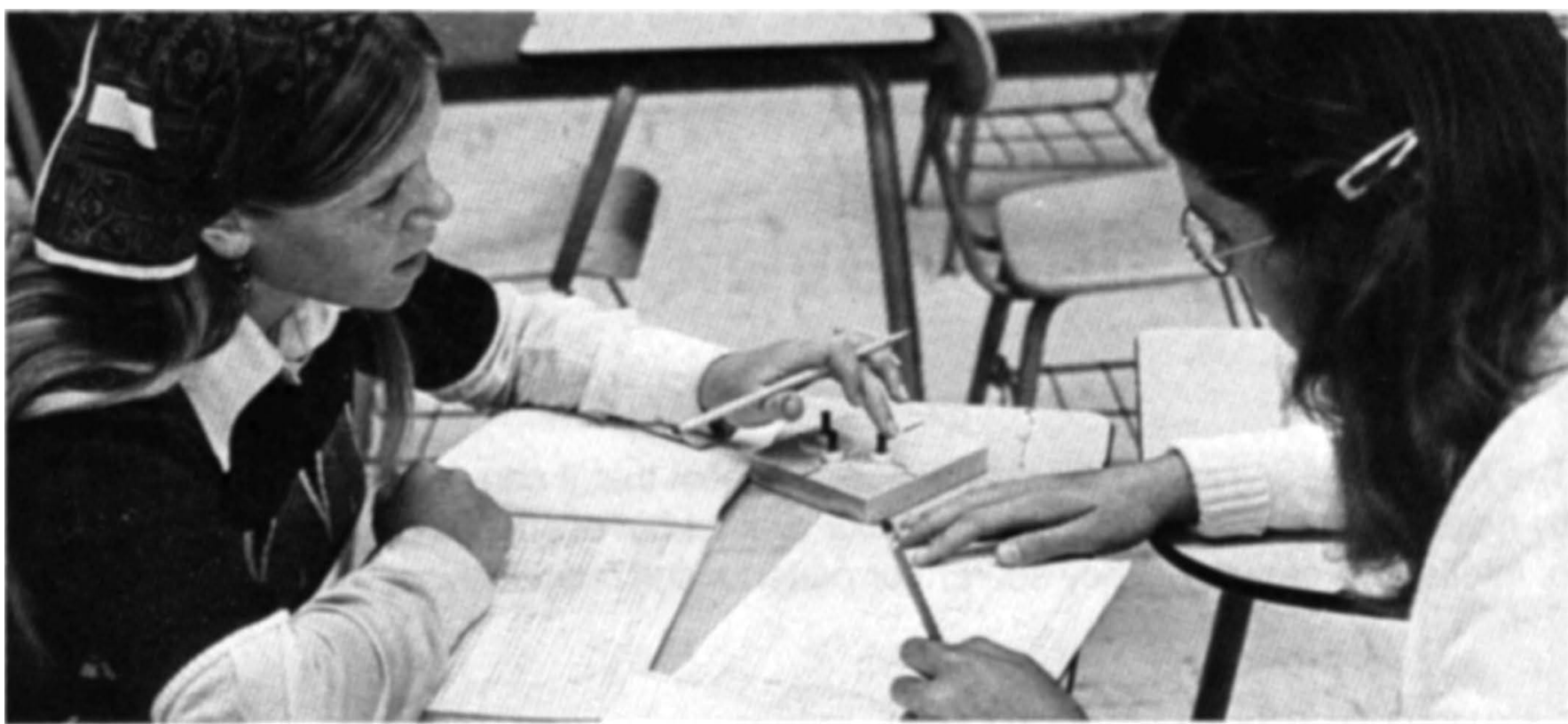

The wheels are turning as these two students compare the angles of rotation of three intermeshing gears. Their search for numerical relationships will help them develop proportional reasoning and understand

when to apply this pattern of thought. The ability to handle functional relationships such as proportionality is a characteristic of formal reasoning, the fourth of Plaget's stages of intellectual development.

direct correspondence between the arrangement of the dots and the physical examples given. Although he introduces the idea of "acceleration," he does not indicate that he has any more than a vague general idea of its meaning. In a similar way, Deloris concentrates on the spatial arrangement of the islands. Her explanations have more to do with her perception of the physical arrangement of the islands than with the clues given in the puzzle. Both Fred and Deloris appear limited in their reasoning to the specific details of a puzzle, and do not readily relate the facts of the puzzles to more general principles.

Consider, on the other hand, the responses of James and Myrna. Both of them have made conjectures to facilitate answering the questions. James, who had not previously used a ticker tape, begins his explanations with generalized concepts such as constant speed, deceleration, acceleration and a frictionless sys. tem. Even when his explanation is wrong ("acceleration is exponential") he demonstrates that he is reasoning within a system of deduction from hypotheses, in which a ticker tape can serve as one specific example representative of a more general principle.

Myrna, as she reasons about the Islands Puzzle, fits the clues into an overall scheme for explaining the air travel between the islands. She suggested a hypothetical trip, demonstrating the correctness of her answer by reasoning to a contradiction. James and Myrna display patterns of reasoning commonly used by physicists.

Even in the responses to these simple written puzzles, the qualitative differences in student reasoning are vividly displayed. For an understanding of these differences, let us turn to the work of $\mathrm{Pi}$ aget.

\section{The development of reasoning}

Jean Piaget began his research on children in about 1920 . The results of his work of primary concern to us are reported in the book, The Growth of Logical Thinking from Childhood to Adolescence. ${ }^{3}$ In this book the responses of young people to various tasks concerning physical phenomena are described. These tasks included physics experiments such as those on the equality of the angles of incidence and reflection, the law of floating bodies, the flexibility of metal rods, the oscillation of a pendulum, the motion of bodies on an inclined plane, the conservation of momentum of a horizontal plane, the equilibrium of a balance and the projection of shadows.

On the basis of the responses, Piaget and his co-workers developed a theory for interpreting the development of what he considers to be universal patterns of reasoning. Pivotal to this theory is the concept of stages of intellectual development. The stages-there are four in the theory - are characterized by distinctive features in the patterns of a person's reasoning. It was hypothesized that each of Piaget's four stages serves as a precursor to all succeeding stages, so that reasoning develops sequentially, always from the less effective to the more effective stage, although not necessarily at the same rate for every individual.

Like a concept in any theory, a stage of intellectual development is a simplification that is helpful in analyzing and interpreting observations, somewhat like a point particle or a frictionless plane in mechanics. In this spirit, we should not expect that most people during their period of development will exhibit all the reasoning characteristics of, say, stage $A$ for a certain period of time and then suddenly change to all the reasoning patterns appropriate to stage B. Rather, the development of a person's reasoning should be thought of as gradual, at a particular time showing the features of stage A on some problems while exhibiting certain features of stage B on others. The stage concept therefore may be more useful for classifying reasoning patterns than for describing the overall intellectual behavior of every particular person at a given time.

The first Piagetian stage is called sensory-motor. This stage is characteristic of children's thinking from birth to about two years of age. Piaget's work with infants provided an explanation for the humor of the "peek-a-boo" game:

The young infant appears to think that the only objects that exist are the objects that can be seen. The sudden "creation" of a large person by removing a blanket covering him does seem to be a funny event. Subsequent experiences provide the child with the opportunity to develop an awareness of the permanence of $\mathrm{ma}$ terial objects.

The concept of permanence provides the basis for the child's need for language. If objects do exist when they are out of sight, then it is useful to have symbols (or words) to represent them. So the sensory - motor stage serves as the precursor for the next, pre-operational, stage.

During the pre-operational period the child is learning words and trying to fit his experiences of the world together. The pre-operational child lives in a very per- 


\section{The ticker-tape puzzle}

The puzzle below is a task designed to display the variety of student reasoning patterns used in a typical physics classroom activity. It is taken from materials for the workshop on Physics Teaching and the Development of Reasoning offered at the 1975 AAPT-APS meeting in Anaheim, California (reference 1).

Start

A

\begin{tabular}{|lllllllllllll}
\hline$\bullet \bullet$ & $\bullet$ & $\bullet$ & $\bullet$ & $\bullet$ & $\bullet$ & $\bullet$ & $\bullet$ & $\bullet$ & $\bullet$ \\
\hline$\bullet$ & $\bullet$ & $\bullet$ & $\bullet$ & $\bullet$ & $\bullet$ & $\bullet$ & $\bullet$ & $\bullet$ & $\bullet$ & $\bullet$ & $\bullet$ \\
\hline
\end{tabular}

B

$\bullet \bullet \bullet-1 \cdot$

C

$+$

D

$\bullet \bullet \bullet \bullet \bullet \bullet \bullet \bullet \bullet \bullet$

E

$\bullet \bullet \bullet \bullet \bullet \bullet \bullet \bullet \bullet \bullet \bullet \bullet \bullet \bullet \bullet \bullet$

Many physics labs allow you to study motion by making timer tapes like the five illustrated above. These are strips of paper attached to a moving object and passing through a timing mechanism that makes a row of small dots by striking regularly at equal time intervals, usually five to ten times per second.

- Have you ever used or watched such a device?

- Identify the tape that fits each of the examples below and justify your answers, taking special care to mention any tapes that a less experienced student might easily mistake for the correct one.

1. A student walking through the laboratory at constant speed

A BCDE Justification?

2. A cart gradually slowing down on a level plane

A B CDE Justification?

3. A cart rolling freely down an inclined plane Justification?

A BCDE

4. Explain how one of the two remaining tapes might have been made, and briefly justify your hypothesis.

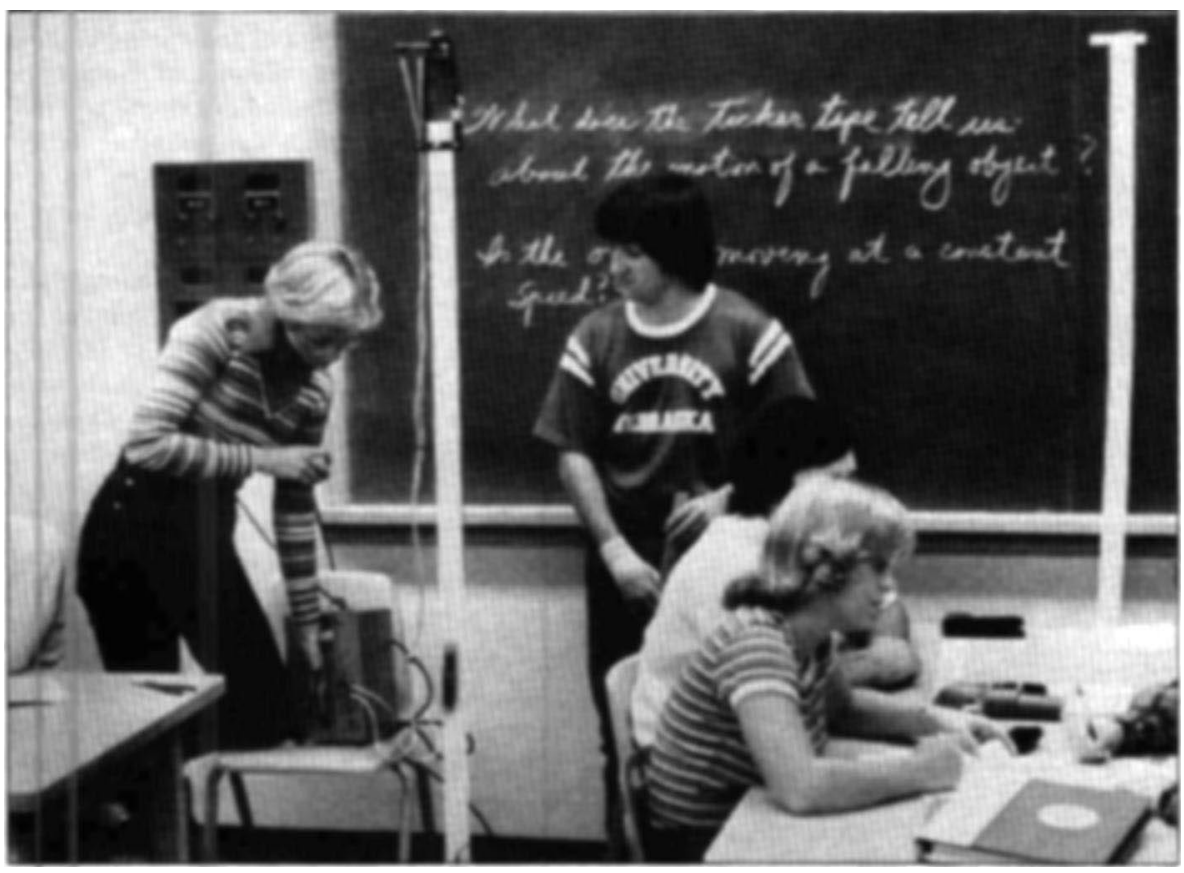

Sparks mark the position of the falling object on the ticker tape. The dot patterns can not be andilysed readily by that third of US adolescents and adults who use only concrete reasoning. sonal world with his own ego at the center ("The Sun is following me!"). He puts facts together to produce ad-hoc explanations, such as, "My dad mows the yard because he's a physicist."

The pre-operational child does not use causal reasoning. Some authors have used children's pre-causal explanations as the motif for humorous books. For Piaget, such explanations are clues as to how children think about the world in which they live.

The first two Piagetian stages are usually completed before a person is nine years old. The child's interaction with physical systems plays an essential role in his or her intellectual development during the first two stages. The role of physics in the development of reasoning in the elementary-school years was discussed in a special issue of PHYSICS TODAY. ${ }^{4}$

\section{Concrete reasoning}

To explain the qualitative differences in the reasoning patterns of older students' responses to the two puzzles described earlier we must look to Piaget's third and fourth stages of intellectual development, concrete reasoning and formal reasoning. Certain characteristics help identify reasoning patterns associated with these two stages.

Here are some of the characteristics of concrete reasoning patterns; illustrative examples are added in parentheses:

Class inclusion A person at this stage understands simple classifications and generalizations of familiar objects or events (can reason that all aluminum pieces can close an electric circuit, but not all objects that close a circuit are made of aluminum).

Conservation Such a person reasons that, if nothing is added or taken away, the amount or number remains the same even though the appearance differs (that when water is poured from a short wide container into a tall narrow container, the amount of water is not changed).

Serial ordering The person arranges a set of objects or data in serial order and may establish a one-to-one correspondence ("The heaviest block of copper stretches the spring the most.").

Reversiblilty A person using concrete reasoning mentally inverts a sequence of steps to return from the final to the initial conditions (reasoning that the removal of weight from a piston will enable the enclosed gas to expand back to its original volume).

Concrete reasoning enables a person to

- understand concepts and simple hypotheses that make a direct reference to familiar actions and objects, and can be explained in terms of simple associations ("A larger force must be applied to move a larger mass.");

- follow step-by-step instructions as in a recipe, provided each step is specified (carry out a wide variety of physics ex- 


\section{The islands puzzle}

The puzzle below is a written task designed to display the variety of deductive-logic strategies used by adolescents (reference 2).

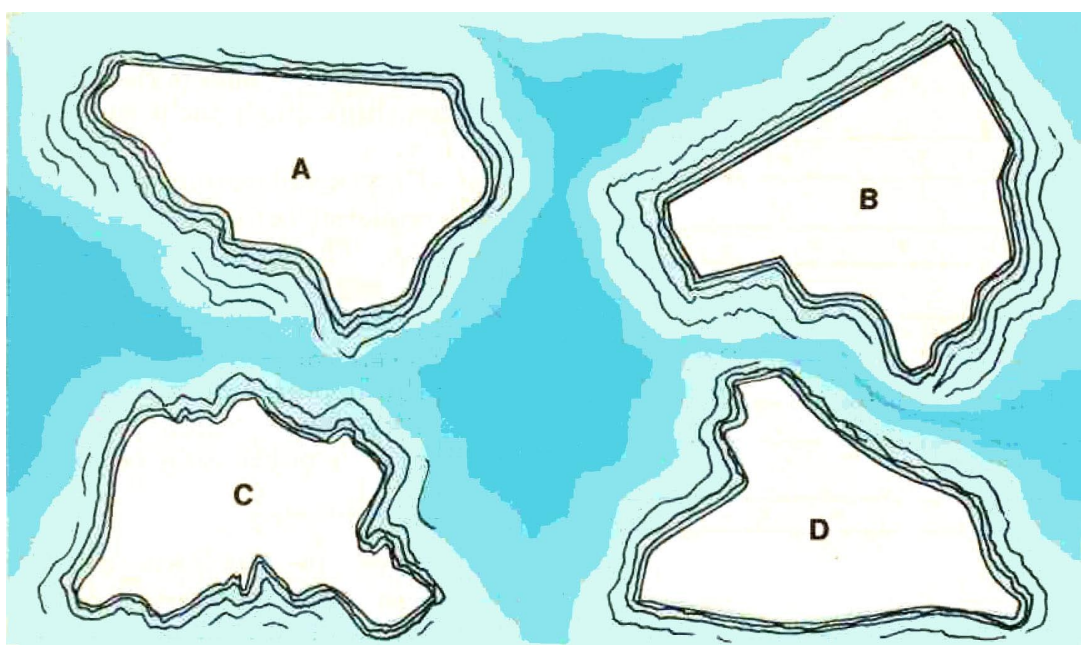

There are four islands in the ocean, islands A, B, C and D. People have been travelling these islands by boat for many years, but recently an airline started in business. Carefully read the clues about possible plane trips at present. The trips may be direct or include stops and plane changes on an island. When a trip is possible, it can be made in either direction between the islands. You may make notes or marks on the map to help use the clues.

First clue: People can go by plane between Islands $C$ and $D$.

Second clue: People can not go by plane between Islands A and B.

- Use these clues to answer Question 1. Do not read the next clue yet.

1. Can people go by plane between Islands B and D?

Yes__ No ___ Can't tell from the two clues___ Please explain your answer. Third clue (do not change your answer to Question 1 now!): People can go by plane between Islands B and D.

- Use all three clues to answer Questions 2 and 3.

2. Can people go by plane between Islands $B$ and $C$ ?

Yes__ No ___ Can't tell from the three clues

Please explain your answer.

3. Can people go by plane between Islands $A$ and $C$ ?

Yes__ No _ _ Can't tell from the three clues_

Please explain your answer. element theory of color vision),

Control of variables In establishing the truth or falsity of hypotheses, a person recognizes the necessity of taking into consideration all the known variables and designing a test that controls all variables but the one being investigated (for example, changing only the direction of the light to detect the possible existence of the ether),

Concrefe reasoning about constructs A person applies multiple classification, conservation, serial ordering and other reasoning patterns to concepts and abstract properties (for example, applying conservation of energy to propose the existence of the neutrino),

Functional relationships A person recognizes and interprets dependencies between variables in situations described by observable or abstract variables, and states the relationships in mathematical form (for example, stating that the rate of change of velocity is proportional to the net force),

Probabilistic correlations A person recognizes the fact that natural phenomena themselves are subject to random fluctuations and that any explanatory model must involve probabilistic considerations, including the comparison of the number of confirming.and disconfirming cases of hypothesized relations (for example, arguing from the small number of alpha particles scattered through large angles from gold foil to suggest a nuclear model for the atom).

Formal reasoning patterns, taken in concert, enable individuals to use hypothesis and deduction in their reasoning. They can accept an unproven hypothesis, deduce its consequences in the light of other known information and then verify empirically whether, in fact, those consequences occur. Furthermore, they can reflect upon their own reasoning to look for inconsistencies. They can check their results in numerical calculations against order-of-magnitude estimates. James and Myrna, in their responses to the puzzles, gave evidence of using formal reasoning.

In the table on page 28 we summarize some differences between reasoning at the concrete and formal levels. It is quite clear that a successful physicist makes use of formal reasoning in his area of profes. sional expertise. In fact, formal reasoning is prerequisite for producing quality work in physics.

Many theoretical and experimental issues relating to Piaget's work are still being investigated. Piaget's original notion was that all persons use formal reasoning reliably by their late teens. Yet. recent studies strongly suggest that, although almost everyone becomes able to use concrete reasoning, many people do not come to use formal reasoning reliably. These persons often appear to be reasoning at the formal level and/or com. prehending formal subject matter when 


\section{Workshops and programs based on Piaget's concepts}

Workshops that focus on physics teaching and the development of reasoning have been offered at professional meetings and on individual college campuses. The workshop materials for examing instructional aids in various subject areas are available from several sources:

- Physics Teaching and the Development of Reasoning Workshop Materials, AAPT Executive Office, Graduate Physics Building, S.U.N.Y., Stony Brook, N.Y. 11794;

- Biology Teaching and the Development of Reasoning Workshop Materials, Lawrence Hall of Science, Berkeley. Cal. 94720;

- Science Teaching and the Development of Reasoning Workshop Materials (includes physics, chemistry, biology, general science and earth sciences), Lawrence Hall of Science, Berkeley, Cal. 94720, and

- College Teaching and the Development of Reasoning Workshop Materials (includes anthropology, economics, English, history, mathematics, philosophy and physics materials), ADAPT, 213 Ferguson Hall, University of Nebraska-Lincoln, Lincoln, Neb 68588.

Another such workshop is being sponsored by the American Association of Physics Teachers at the joint APS-AAPT meeting in 'Chicago this month.

College students are being encouraged to develop their reasoning in several programs, including:

physical-science programs, such as those led by Arnold B. Arons, University of Washington (Amer. J. Phys. 44, 834; 1976) and John W. Renner, University of Oklahoma (Amer. J. Phys. 44, 218; 1976);

the introductory physics laboratory course for engineering students developed by Robert Gerson, University of Missouri-Rolla, and

- two Piaget-based multidisciplinary programs for college freshmen, ADAPT at the University of Nebraska-Lincoln and DOORS at Illinois Central College, East Peoria.

they are actually only applying memorized formulas, words or phrases.

The development of formal reasoning represents an extremely worthwhile educational aim. Formal reasoning is fundamental to developing a meaningful understanding of mathematics, the sciences and many other subjects of modern life. The finding, by a wide variety of studies, ${ }^{5}$ that more than one third of the adolescents and adults in the United States do not employ formal reasoning patterns effectively presents a real educational challenge. What can be done about the significant fraction of the population that appears to be stuck at the stage of concrete reasoning?

\section{Self-regulation}

As physicists, we can see the advantages to our profession of more widespread use of formal reasoning patterns. To see the role that physics would have to play in creating the necessary atmosphere

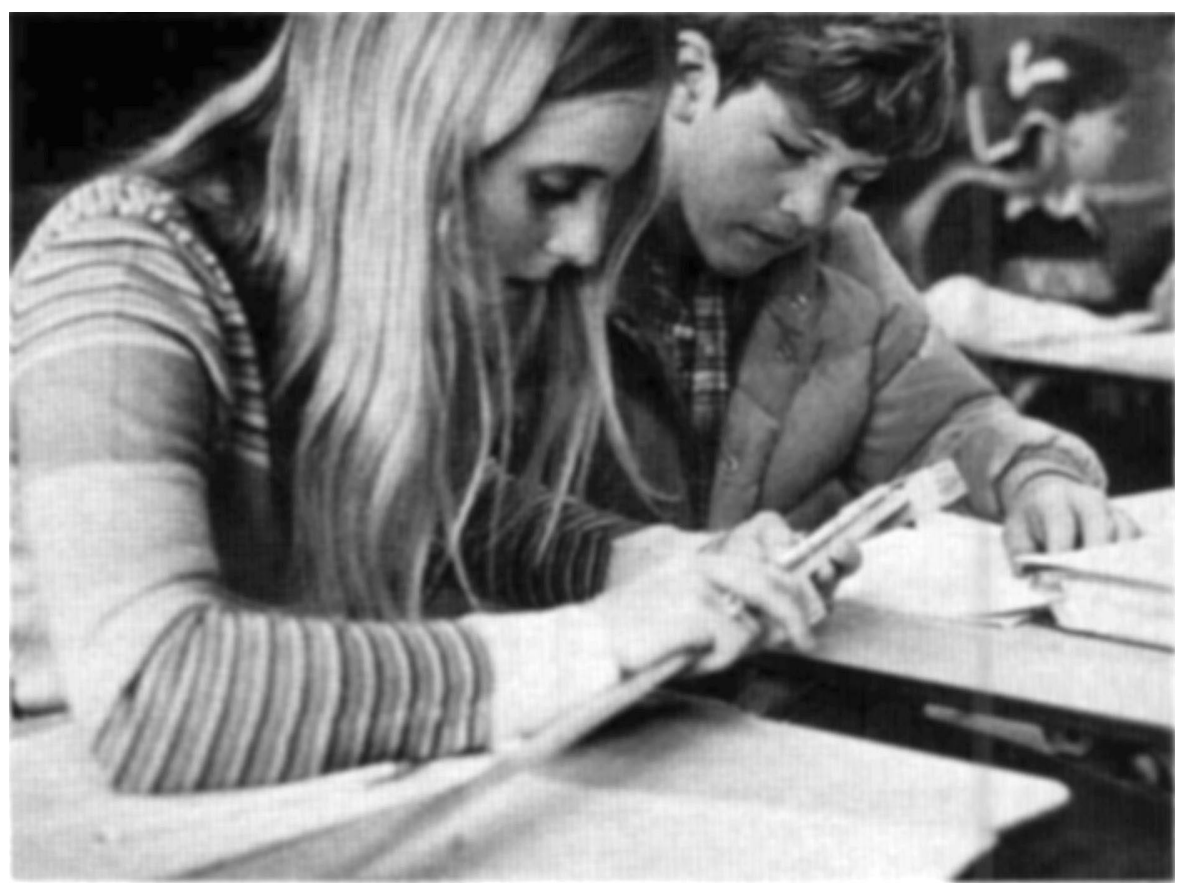

By comparing the extensions of a coil spring at various points, these students are gaining insight into proportionality; such formal-reasoning patterns are attained through self-regulation.

for this, let us turn to another concept in Piaget's theory of intellectual development, that of self-regulation.

Self-regulation is the process whereby an individual's reasoning advances from one level to the next, an advance that is always in the direction toward more successful patterns of reasoning. Piaget considers this process of intellectual development as analogous to the differentiation and integration one sees in the biological development of an embryo, as well as analogous to the adaptation of evolving species.

A person develops formal reasoning only through the process of self-regulation. Concrete reasoning thus is a prerequisite for the development of formal reasoning.

The process of self-regulation is one in which a person actively searches for relationships and patterns to resolve contradictions and bring coherence to a new set of experiences. Implicit in this notion is the image of a relatively autonomous person, one who is neither under the constant guidance of a teacher nor strictly bound to a rigid set of precedents.

Self-regulation can be described as unfolding in alternating phases, beginning with assimilation. The individual's reasoning assimilates a problem situation and gives it a meaning determined by present reasoning patterns. This meaning may or may not, in fact, be appropriate. Inappropriateness produces what is called "disequilibrium," "cognitive conflict" or "contradiction," a state that, according to Piaget, is the prime mover in initiating the second phase-accomodation.

Accomodation entails
- an analysis of the situation to locate the source of difficulty and

- formation of new hypotheses and plans of attack.

Just how this is done varies from person to person and depends upon his analytical and problem-solving abilities. The results of these reflective and experimenting activities are new reasoning patterns that may include new understandings. In terms of assimilation and accommodation, self-correcting activities (accommodation) are constantly being tested (assimilation) until this alternation of phases produces successful behavior. The whole self-regulation process, directed at a stable rapport between patterns of reasoning and environment, is often called "equilibration" by Piaget.

Recall the self-regulation process that Count Rumford recounts in his essays on heat. ${ }^{\text {' }}$ In Piaget's terms, Rumford experienced cognitive conflict by the extraordinary ability of apple pies to retain their heat, by the fact that heat had no effect upon the weight of objects and by the intense heat of the metallic chips separated from the cannons he bored. He could not assimilate these experiences with the caloric theory of heat, so he rejected that theory. He accommodated his reasoning to experience by developing the idea that heat was excited and communicated by motion.

The development of reasoning has two requirements: Exploratory experiences with the physical world, and discussion and reflection upon what has been done, what it means and how it fits, or does not fit, with previous patterns of thinking. This suggests that experiences gained through physics can play a key role in the 
development of reasoning and understanding.

\section{Role of the physics community}

Let us examine how physics could be used to foster self-regulation in a person. Two factors appear to be required:

- He must be faced with a physical situation that he can only partially understand in terms of old ideas and

- he must have sufficient time to grapple mentally with the new situation, possibly with appropriate hints, but without being told the answer - people must be allowed to put their ideas together for themselves.

The ideal situation would be one in which the problems experienced are felt to be solvable. The Piaget hypothesis is that a challenging but solvable problem will place persons into an initial state of disequilibrium. Then, through their own efforts at bringing together this challenge with their past experiences and what they learn from teachers or peers, they will gradually reorganize their thinking and solve the problem successfully. This success will establish a new and more stable equilibrium with increased understanding of the subject matter and increased problem-solving capability, that is, intellectual development.

One example of such a use of physics is an exhibit of a spring scale and an equal. arm balance mounted on the wall of an elevator in a public building. ${ }^{7}$ The riders in the elevator noticed that the "weight" of the object on the scale varied while the balance remained stationary, a paradox that gave rise to some cognitive conflict. A small card beside the exhibit asked questions and offered hints to encourage the riders to accomodate to this experience.

Physics programs, done properly, can be effective means of promoting intellectual development. Such developmental-physics programs are not aimed at producing more physicists, but at enabling people to develop their potential for formal reasoning. This reasoning can serve them well in many aspects of our technological society.

If physics is an essential element in the growth of reasoning, why are persons so turned off by physics? It seems to us that the physics community has chosen to isolate itself from individuals using primarily concrete reasoning patterns. It has been suggested that all of the junior and senior high-school physics curricula that have been developed in the last 25 years have been intended for students who typically use formal reasoning.

True, modern secondary-school physics courses, such as PSSC Physics and the Project Physics course, have directed students toward laboratory experiments. Yet many of the experiments can only be understood within the hypothetical structure of the formal laws of physics. For example, the use of stroboscopic

\section{Concrete versus formal reasoning}

\section{In concrete reasoning, a person}

- needs reference to familiar actions, objects and observable properties;

- uses classification, conservation, serial ordering and one-to-one correspondence in relation to concrete items above;

- needs step-by-step instructions in a lengthy procedure, and

- is not aware of his own reasoning, inconsistencies among various statements or contradictions with other known facts.

\section{In formal reasoning, a person}

- can reason with concepts, relationships, abstract properties, axioms and theorles;

- uses symbols to express ideas:

- applies combinatorial, classification, conservation, serial ordering and proportional reasoning in these abstract modes of thought;

- can plan a lengthy procedure to attain given overall goals and resources, and

is aware of and critical of, his own reasoning, and actively checks on the validity of his conclusions by appealing to other information.

From Module 9 of the Science Teaching and the Development of Reasoning workshop materials (see the Box on page 27).

photographs to analyze the collisions of two objects appear to be at least as demanding as the Ticker-Tape Puzzle; yet we have seen that the solution to the Ticker-Tape Puzzle was inaccessible to students who used only concrete reasoning.

In short, our fixation on the formal aspects of physics instead of its concrete experiences has made physics unnecessarily difficult and dry. We have removed the sense of exploration and discovery from the study of physics for the majority of students. Several generations of public-school students have been alienated from physics. 8.9

What can you do to make the study of physics less a slave to the formal structure of the discipline and more of a servant to the development of reasoning? You can

- become more familiar with the appli. cations of Piaget's ideas to learning from physics;

- learn about the present attempts to offer Piaget-based programs for large numbers of students;

- encourage your school or college to initiate some programs that focus on the development of reasoning rather than the mastery of content;

- assist service clubs and other groups to present physics to the citizens by means of displays, exhibits and media, and

- develop your skills as a facilitator of self-regulation in others. ${ }^{10}$

The Box on page 27 lists some sources of workshop materials, as well as current college programs based on the Piaget concepts.

\section{The human potential}

As a result of our professional experiences, we of the physics community may possess a valuable insight: that carefully planned interactions of persons with the experimental systems and concepts of physics can contribute vitally to the full human potential. Perhaps our efforts to increase the appropriate people-physics interactions are as important to the future of mankind as our continuing efforts to increase our fundamental understanding of physical systems.

This material is based upon work done as a part of AESOP (Aduancing Education through Science-Oriented Programs), supported by the US National Science Foundation under Grant No. SED74-18950. The opinions are those of the authors and do not necessarily reflect the views of the froundation.

\section{References}

1. Proceedings of the Workshop of Physics Teaching and the Development of Reasoning (Anaheim, Calif. January 1975), American Association of Physics Teachers, Stony Brook, N.Y. (1975).

2. E. F. Karplus, R. Karplus, School Sci. and Math. 70, 5(1970).

3. B. Inhelder, J. Piaget, The Growth of Lonical Thinking from Childhood to Adolescence, Basic Books, New York (1958).

4. PHYSIC'S TODAY, June 1972.

5. D. Griffiths, Amer. J. Phys. 14, 81 (1976); G. Kolodiy, J. Coll. Sci. Teach. 5, 20 (1975); A. E. Lawson, F. Nordland, A. DeVito, J. Res. Sci. Teach. 12, 423 (1976); J.W. McKinnon, J. W. Renner, Amer. J. Phys. 39, 1047 (1971); J. W. Renner, A. E. Lawson, Phys. Teach. 11, 273 (1973); C. A. Tomlinson-Keasey, Dev. Psychol. 6, 364 (1972).

6. The Collected Works of Count Rumford (S. C. Brown, ed.). Harvard U. P., Cam. bridge, Mass. (1968).

7. L. Eason, A. J. Friedman, Phys. Teach. 13, 491 (1975).

8. P. de H. Hurd, School Sci, and Math. 53, 439 (1953).

9. M. B. Rowe, The Science Teacher 42, 21 (1975).

10. A. B. Arons, Amer. J. Phys. 44, 834 (1974). 